\title{
Manejo de las atribuciones de causalidad en un caso de tricotilomanía
}

Cecilia Méndez Sánchez ${ }^{1}$

\section{Introducción}

La tricotilomanía (TTM) es un trastorno con alta incidencia en población infantil entre los 5 y los 13 años. Se estima que su prevalencia oscila entre el 0.5 a $3.4 \%$ en promedio, llegando incluso hasta $13 \%$ de los escolares (Jiménez, Petrocelli y Hernández, 2010) y suele mantenerse durante mucho tiempo o bien aparecer y desaparecer en distintos momentos de la vida de la persona.

La Tтм se presenta en distintas zonas del cuerpo, siendo las más frecuentes la cabeza, las cejas y las pestañas. Suele darse en estados de estrés, aunque se ha visto que muchas personas lo hacen en estados de relajación o cuando están distraídos, luego de experimentar una sensación de hormigueo. Muchos comentan que en su intento de resistirse a la "necesidad" de arrancarse el pelo, experimentan un incremento de la tensión y luego una sensación de descanso al habérselo arrancado, con

1 Universidad Autónoma de Aguascalientes. 
la desaparición de la sensación de hormigueo acompañado de prurito en las áreas afectadas.

La TTM está asociada a comportamientos como morderse las uñas, rascarse, hacerse excoriaciones, tricofagia (entre los que se encuentra el tricobezoar y el síndrome de Rapunzel), la cual puede provocar problemas físicos graves para la persona (anemia, dolor abdominal, náuseas y vómitos, obstrucción e incluso perforación abdominal). Sus principales consecuencias psicológicas son la evitación de situaciones sociales para ocultar la alopecia (Christenson y Mansueto, 1999; Morales, Camacho, Coll y Vázquez, 2010; Stemberger, Thomas, Mansueto y Carter, 2000) y un deterioro de la autoestima, por lo que resulta importante realizar un diagnóstico oportuno para implementar un procedimiento terapéutico encaminado a la eliminación de este trastorno.

Los distintos procedimientos utilizados para la intervención en estos casos tienden a referir procedimientos farmacológicos, procedimientos cognitivo conductuales o bien una combinación de ambos (Jiménez, Petrocelli y Hernández, 2010). Los procedimientos cognitivo conductuales han mostrado ampliamente resultados satisfactorios en el tratamiento de la TтM mediante el uso de estrategias como la relajación, el fortalecimiento de conductas incompatibles y la inversión del hábito (Duke, Keeley, Geffken y Storch, 2010; Pérez, 2004; Elliott y Fuqua, 2000; Himle, Flessner, y Woods, 2004). En estos tratamientos se ha observado que los pacientes muestran una tendencia a evitar el tratamiento como una forma de ocultar su trastorno debido a la vergüenza que experimentan (Diefenbach, Tolin, Hannan, Crocetto y Worhunsky, 2005; Shusterman, Feld, Baer y Keuthen, 2009), o bien, porque están convencidos de que "no pueden hacer nada para controlarse" (Walsh y McDougle, 2001).

Dados estos sentimientos de vergüenza e ideas sobre la imposibilidad de controlarse, planteamos que se puede intervenir desde el modelo de las atribuciones de causalidad de B. Weiner, quien postula que las personas ante una situación de fracaso buscan identificar las causas de ese resultado (atribuciones de causalidad), de las cuales se derivan no sólo sus reacciones 
emocionales sino también aspectos cognitivos, motivacionales y conductuales (1986; 1992). Según él, las causas pueden clasificarse en función de tres dimensiones: locus de causalidad, grado de estabilidad y controlabilidad, las cuales tienen consecuencias sobre el comportamiento del sujeto en tres aspectos específicos, a nivel de las expectativas, de la motivación de logro y de las reacciones emocionales.

Desde la dimensión locus de causalidad, las causas pueden ser internas y externas y tiene consecuencias directas sobre la autoestima, las emociones (orgullo, vergüenza y culpa), en las expectativas y en la motivación de logro (Weiner, 1986 y 1991). La dimensión grado de estabilidad se refiere a que las causas se mantienen en el tiempo o en las situaciones; sus efectos se centran principalmente en las expectativas de logro que el individuo puede generar ante situaciones similares, lo que redundará en sentimientos de esperanza/desesperanza, que los lleva a persistir o no en la tarea. Finalmente, en la dimensión controlabilidad, se analiza el grado de control que posee la persona para modificar las causas a las que atribuye el resultado de su comportamiento, con consecuencias en las reacciones emocionales dirigidas hacia uno mismo; en el caso del fracaso sobrevienen reacciones emocionales de culpa e ira debido a la implicación de responsabilidad acompañadas de evaluaciones negativas para el futuro (Rudolph, Roesch, Greitemeyer y Weiner, 2004). Los investigadores coinciden en que el estilo educativo de los padres y las influencias de profesores, grupos de iguales, cultura, etcétera, son determinantes en las formas en que se aprende a realizar las atribuciones de causalidad, así como la motivación de logro (Shaffer, 2002), en la explicación del éxito académico, problemas emocionales y conductuales, responsabilidad social y empatía.

El estudio de las atribuciones de causalidad ha tenido gran influencia en el campo clínico, pero hasta el momento no se ha encontrado un trabajo que refiera la intervención de la тTM bajo la óptica de este modelo. 


\section{Planteamiento del caso}

El caso clínico que se presenta fue intervenido bajo esta óptica y se refiere a una niña de 8 años de edad (tercero de primaria), que vive sólo con su madre de 36 años (empleada de ventas). La niña dejó de ver a su padre desde que tenía 4 años, actualmente rara vez lo ve. Dice que lo extraña mucho y no pierde la esperanza de que "sus padres se casen y se junten". Comenzó a quitarse el cabello detrás de la oreja y continuó con el de la frente, cejas y pestañas desde hacía mes y medio con relación a la primera entrevista con la madre, fecha que coincidió con la entrada a la escuela. Había sido llevada con el psiquiatra, el cual le recetó Avantal (1/2 pastilla al día). La madre dejó de darle el medicamento porque no vio ningún efecto en su hija después de que lo tomó por dos semanas. No se presentaba tricofagia; sin embargo, se acompañaba de la siguiente conducta: morder la parte inferior de la manga de su suéter y/o blusa.

Cuando la niña salía de su escuela, era llevada al lugar de trabajo de la madre (en la parte de atrás del espacio de trabajo de su madre), donde hacía sus tareas. La madre comentó que cuando llegaban a su casa (a las 18:00 horas aproximadamente), ella se dedicaba a las labores del hogar, mientras que la niña miraba la televisión. Comentó que buscaba que su hija tuviera buenas calificaciones y que la niña era muy sensible a las presiones escolares respecto al buen aprovechamiento académico, así como al rechazo social por parte de sus compañeros. Refirió que cuando su hija tenía cuatro años comenzó a comerse las uñas, lo que ha disminuido con el tiempo, aunque no se ha eliminado. Se describe a la niña como callada, le "da pena saludar a las personas" y tiende a decir que cuando lo haga "se va a avergonzar", suele además compararse con los demás y en esa comparación "ella siempre sale perdiendo".

En las sesiones, la niña reporta que se pone nerviosa porque piensa que "se van a burlar de mí". Cada vez que está nerviosa, comienza a rascarse o jalarse el pelo, y siente un dolor ligero pero ya sin ansiedad, y continúa jalándoselo hasta que se cae, esto se acompaña de bienestar. Dice que chupa las co- 
sas porque siente "cosa", y así es como se relaja. Ante algunas situaciones que ve, ella se imagina o piensa cosas donde hay consecuencias negativas y que no puede controlar, entonces experimenta ansiedad y es cuando comienza con el comportamiento-problema. Comenta también que "mis manos me controlan". Refiere que esto sucede principalmente en la escuela, pues le preocupa no hacer bien los trabajos porque se quedaría sin recreo y que las actividades escolares son "difíciles" para ella y eso la pone nerviosa (cuando la maestra regaña a sus compañeros ella piensa que es a ella, así como cuando le preguntan y no sabe la respuesta).

En primera instancia se buscó identificar las atribuciones de causalidad que la niña y la madre tenían sobre la TTM. Posteriormente, en diversas situaciones de juego, se identificaron sus atribuciones de causalidad ante la resolución de problemas y, una vez obtenido el diagnóstico, el cual fue entregado a la madre y a la niña por separado, se procedió con la fase de tratamiento, cuyo objetivo fundamental era fortalecer su percepción de control de las situaciones que le generaban estrés, mediante la sustitución de sus atribuciones de causalidad externas, estables y sin control a atribuciones internas, no estables y con control. A la madre se le instruyó para que modificara su estilo educativo y a que apoyara en casa en el fortalecimiento de las atribuciones de causalidad que se estaban favoreciendo con su hija en el consultorio, así como para que dedicara tiempo para jugar con su hija.

Durante todo el proceso de intervención se tomaron fotografías de las partes afectadas (cabeza, cejas y pestañas), con la finalidad de utilizarlas como un elemento de retroalimentación semanal para la niña y su madre. La fase de seguimiento se realizó al mes, los tres meses y a los seis meses después de finalizado el tratamiento.

La niña logró aceptar que ella tenía el control del estrés que experimenta en distintas situaciones lúdicas durante las sesiones y pudo ser capaz de implementar distintas formas para controlarse. Se pudo constatar en las fotografías tomadas que para la sesión seis le comenzaba a crecer el pelo en la nuca y 
para la sesión ocho le seguía creciendo en la parte frontal de la cabeza. Para la sesión 10 ya tenía pelo en toda la cabeza, así como en cejas y pestañas.

\section{Conclusiones}

Cuando una persona se convence de que es incapaz de controlar su comportamiento y sus emociones, se fortalece la idea de que no tiene control sobre sí misma y de que son las situaciones las que la manejan; en este sentido, las causas de su comportamiento son externas y sin control por parte de ella. Pero cuando la persona cambia sus atribuciones de causalidad, en este caso a causas internas y controlables por ella, se fortalece su autoestima al no verse como vulnerable, su autoconfianza se fortalece y por ende disminuyen los sentimientos de ansiedad; así, la persona confía en su capacidad de controlar su comportamiento, permitiéndose generar formas alternativas adecuadas de afrontamiento de las situaciones que le generaban estrés. Con este caso se ha podido demostrar que el manejo de las atribuciones es una buena alternativa de tratamiento para la TTM.

\section{Referencias}

Christenson, G.A. y Mansueto, C.S. (1999). Trichotillomania: Descriptive Characteristics and Phenomenology. En D.J. Stein, G.A. Christenson y E. Hollander (Eds.), Trichottilomania (1-42). Washington: American Psychiatric.

Diefenbach, G.J., Tolin, D.F., Hannan, S., Crocetto, J. y Worhunsky, P. (2005). Trichotillomania: Impact on Psychosocial Functioning and Quality of Life. Behaviour Research and Therapy, 43, 869-884.

Duke, D.C., Keeley, M.L., Geffken, G.R. y Storch, E.A. (2010). Trichotillomania: a current review. Clinical Psychology Review, 30, 181-193. 
Elliott, A.J. y Fuqua, R.W. (2000). Trichotillomania: Conceptualization, Measurement, and Treatment. Behavior Therapy, 31, 529-546.

Himle, M.B., Flessner, C.A. y Woods, D.W. (2004). Advances in the Behavior Analytic Treatment of Trichotillomania and Tourette's Syndrome. Journal of Early and Intensive Behavioral Intervenctions, 1(1), 58-65.

Jiménez, H.F., Petrocelli, C.D. y Hernández, Z.S. (2010). Caso Clínico. Tricotilomanía. Revista del Centro Dermatológico Pascua, 19(1), 22-26. Recuperado de http://www.medigraphic.com/pdfs/derma/cd-2010/cd101e.pdf.

Morales-Fuentes, B., Camacho-Maya, U., Coll-Clemente, F. y Vázquez-Minero, J. (2019). Tricotilomanía, tricobezoar de repetición y síndrome de Rapunzel. Informe de un caso y revisión de la literatura. Cirugía y Cirujanos, 78(3), 265268.

Pérez, J.M. (2004). Tratamiento conductual de un caso crónico y recidivante de tricotilomanía. Revista Psicología Científica. com, 6(1). Recuperado de http://www.psicologiacientifica. com/tricotilomania-tratamiento-conductual.

Rudolph, U.; Roesch, S.C.; Greitemeyer, T. y Weiner, B. (2004). A Meta-Analytic Review of Help Giving and Aggression From an Attributional Perspective: Contributions to a General Theory of Motivation. Cognition and Emotion, 18(6), 815-848.

Shaffer, D.R. (2002). Logro. Desarrollo social de la personalidad. Madrid: Thomson.

Shusterman, A., Feld, L., Baer, L. y Keuthen, N. (2009). Affective Regulation in Trichotillomania: Evidence from a Large-Scale Internet Survey. Behaviour Research and Therapy, 47, 637-644.

Stemberger, R.M., Thomas, A.M., Mansueto, C.S. y Carter, J.G. (2000). Personal Toll of Tricotillomania: Behavioral and Interpersonal Sequelae. Journal of Anxiety Disorders, 14(1), 97-104.

Walsh, K.H. y McDougle, C.J. (2001). Trichotillomania. Presentation, Etiology, Diagnosis and Therapy. American Journal of Dermatology, 2, 327-333. 
Weiner, B. (1986). An Attributional Theory of Motivation and Emotion. Nueva York: Springer-Verlag.

Weiner, B. (1991). Metaphors in Motivation and Attribution. American Psychologist, 46, 921-930.

Weiner, B. (1992). Human Motivation: Metaphors, Theories, and Research. Newbury Park, cA: Sage Publications. 